\title{
Wastewater treatment plant resistomes are shaped by bacterial composition, genetic exchange, and upregulated expression in the effluent microbiomes
}

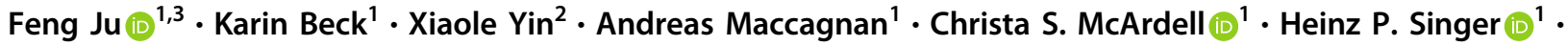 \\ David R. Johnson $\mathbb{1}^{1} \cdot$ Tong Zhang ${ }^{2} \cdot$ Helmut Bürgmann $\mathbb{1}^{1}$
}

Received: 28 March 2018 / Revised: 19 August 2018 / Accepted: 26 August 2018 / Published online: 24 September 2018

(c) International Society for Microbial Ecology 2018

\begin{abstract}
Wastewater treatment plants (WWTPs) are implicated as hotspots for the dissemination of antibacterial resistance into the environment. However, the in situ processes governing removal, persistence, and evolution of resistance genes during wastewater treatment remain poorly understood. Here, we used quantitative metagenomic and metatranscriptomic approaches to achieve a broad-spectrum view of the flow and expression of genes related to antibacterial resistance to over 20 classes of antibiotics, 65 biocides, and 22 metals. All compartments of 12 WWTPs share persistent resistance genes with detectable transcriptional activities that were comparatively higher in the secondary effluent, where mobility genes also show higher relative abundance and expression ratios. The richness and abundance of resistance genes vary greatly across metagenomes from different treatment compartments, and their relative and absolute abundances correlate with bacterial community composition and biomass concentration. No strong drivers of resistome composition could be identified among the chemical stressors analyzed, although the sub-inhibitory concentration (hundreds of $\mathrm{ng} / \mathrm{L}$ ) of macrolide antibiotics in wastewater correlates with macrolide and vancomycin resistance genes. Contig-based analysis shows considerable colocalization between resistance and mobility genes and implies a history of substantial horizontal resistance transfer involving human bacterial pathogens. Based on these findings, we propose future inclusion of mobility incidence (M\%) and host pathogenicity of antibiotic resistance genes in their quantitative health risk ranking models with an ultimate goal to assess the biological significance of wastewater resistomes with regard to disease control in humans or domestic livestock.
\end{abstract}

\section{Introduction}

Anthropogenic release of antibiotic resistance genes (ARGs) into environmental reservoirs has raised global

Electronic supplementary material The online version of this article (https://doi.org/10.1038/s41396-018-0277-8) contains supplementary material, which is available to authorized users.

Helmut Bürgmann

helmut.buergmann@eawag.ch

1 Eawag, Swiss Federal Institute of Aquatic Science and Technology, 6047 Kastanienbaum or 8600 Duebendorf, Switzerland

2 Department of Civil Engineering, Environmental Biotechnology Lab, The University of Hong Kong, Hong Kong 999077, China

3 Present address: Center for Environmental and Resources Engineering, Institute of Advanced Technology, School of Engineering, Westlake University, Hangzhou 310064, China public health concerns $[1,2]$. The importance of wastewater treatment plants (WWTP) both as a barrier for resistant bacteria and as a potential hotspot for dissemination has been highlighted, although the evaluation of the risks for human health remains unresolved [3, 4]. The increasing environmental occurrence of clinically relevant ARGs and evidence for horizontal dissemination of resistance between environmental bacteria and human pathogens demonstrate the importance of environmental resistomes (collections of resistance genes in a metagenome) [1,5-7]. Communal WWTPs receive diverse anthropogenic antimicrobial and microbiological contaminants including antibiotics [8], biocides [9], metals [10], and human pathogens [11]. Metagenomic or qPCR analysis of genomic or plasmid DNA highlight the (co-)occurrence and prevalence of diverse ARGs and metal resistance genes (MRGs) in WWTPs [12-17], which are implicated as point sources for their release into the environment [16, 18]. Moreover, several PCR-based and cultivation-based studies have 
a) WWTP compartments are contrasting habitats

b) Changing stressor concentrations
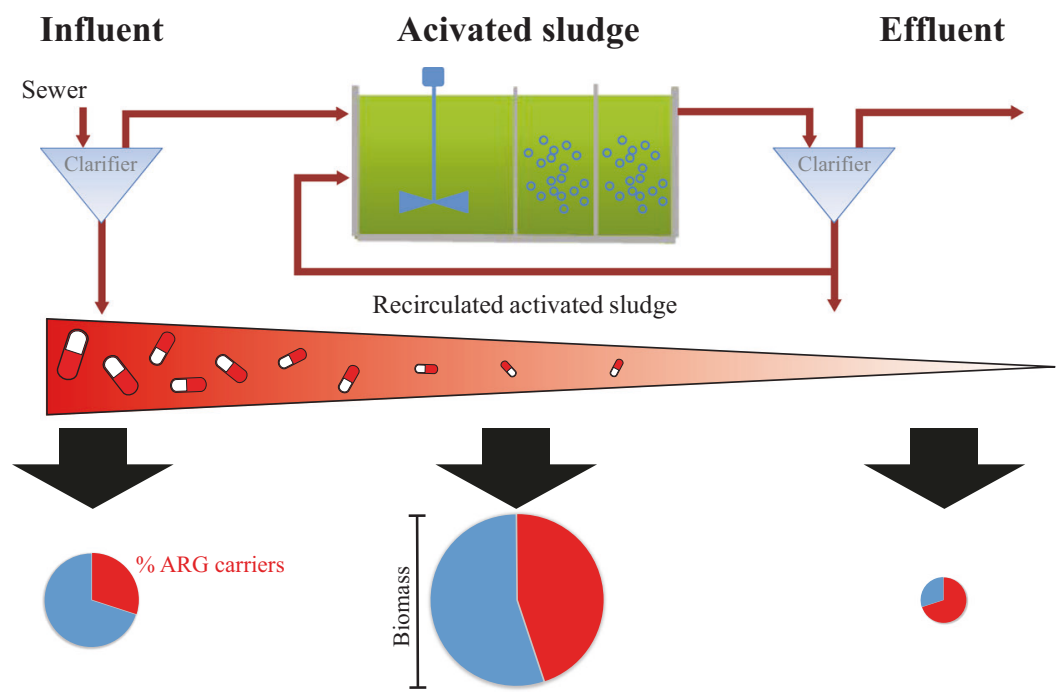

d) Community shift

c) Biomass change and enrichment of ARG
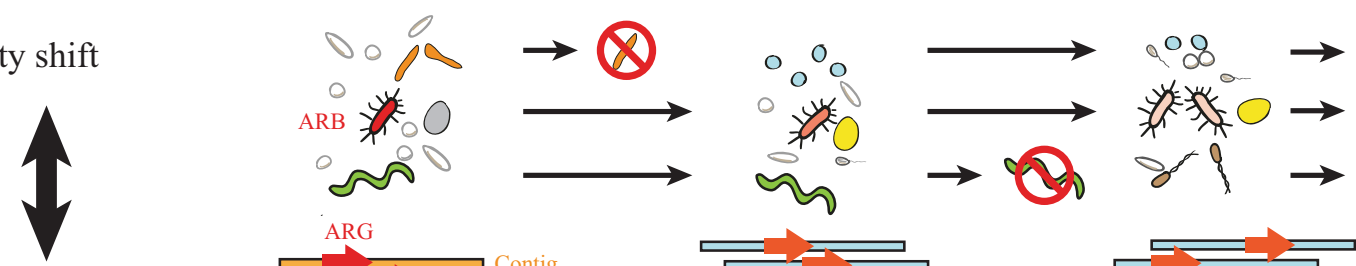

e) Resistome shift
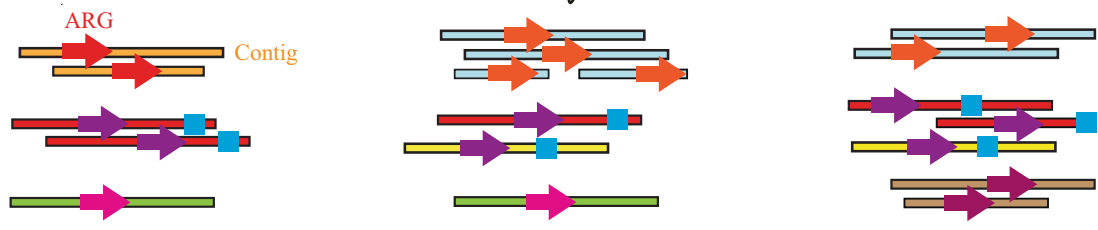

f) Horizontal gene transfer
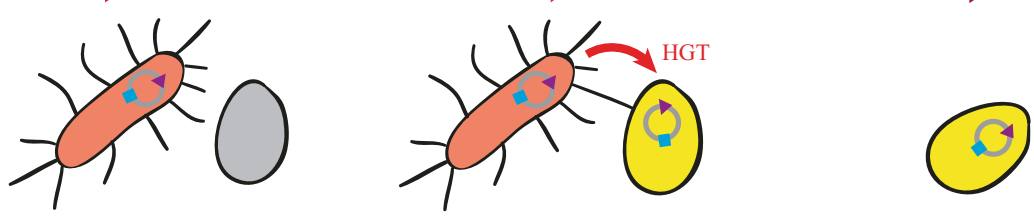

Fig. 1 Key hypotheses about processes affecting the resistome (resistance gene content of the microbial metagenome) during passage of a WWTP. The WWTP consists of compartments with contrasting environmental conditions (a) including changing concentrations of antibiotics, metals, and other stressors (b) that may act as drivers on microbial community assembly and resistomes. By design (activated sludge process), and as an effect of the changing habitat conditions, we expect changes in biomass per volume (piechart area) but also persistence or even enrichment of ARG-carrying bacteria (red wedge) (c). Likewise, we expect a strong shift in the composition of the microbial community as a whole (d), and the antibiotic-resistant subset (colored, $\mathrm{ARB}$ ). These changes are expected to correlate to changes of the resistome (e), which are here shown as metagenomic contigs (bars colored by bacterium of origin) carrying different ARGs (colored arrows). ARB and ARGs discharged with the effluent may have different origins: Some may have passed through the entire WWTP if the bacteria survive treatment (here, e.g., the red bacterium), others may originate from populations of bacteria that grow in the WWTP (blue

and brown bacteria). If the environmental conditions in the WWTP favor populations that carry ARGs, these ARGs may become enriched in the bacterial community of the effluent. Studying ARG transcription and changes of transcription across stages (indicated by different shades of the red bacterium (d)) may provide clues if genes that are enriched are also active. A contig-centered analysis further allows identification of marker genes for mobile genetic elements (blue squares) occurring on the same contig as an ARG. Horizontal gene transfer may act on evolutionary timescales (f), thus that, e.g., resistance plasmids arriving with human pathogens or commensals in the inflow could eventually become established also in WWTP bacteria. If horizontal transfer of ARGs would happen with such high frequency that it amounts to a mass flow on timescales relevant to the flow of biomass, shifts in the population size of the original host bacteria may no longer correlate with ARG abundance, and the resistome structure could shift independently of the phylogenetic community structure (color figure online)

detected vancomycin-resistant enterococci, methicillinresistant staphylococci, and cefazolin-resistant Enterobacteriaceae in wastewater biofilm, as well as clinically relevant ARGs (e.g., CTX-M, ampC, qnr, and NDM-1) in the final effluent $[6,19,20]$. However, the mechanisms which allow resistance genes to traverse WWTPs and how

they are influenced by secondary treatment remain open questions (Fig. 1).

Environmental contaminants including metals and biocides represent widespread and recalcitrant stressors in the WWTP environment that might exert selective pressure that potentially contribute to the persistance and enrichment of 


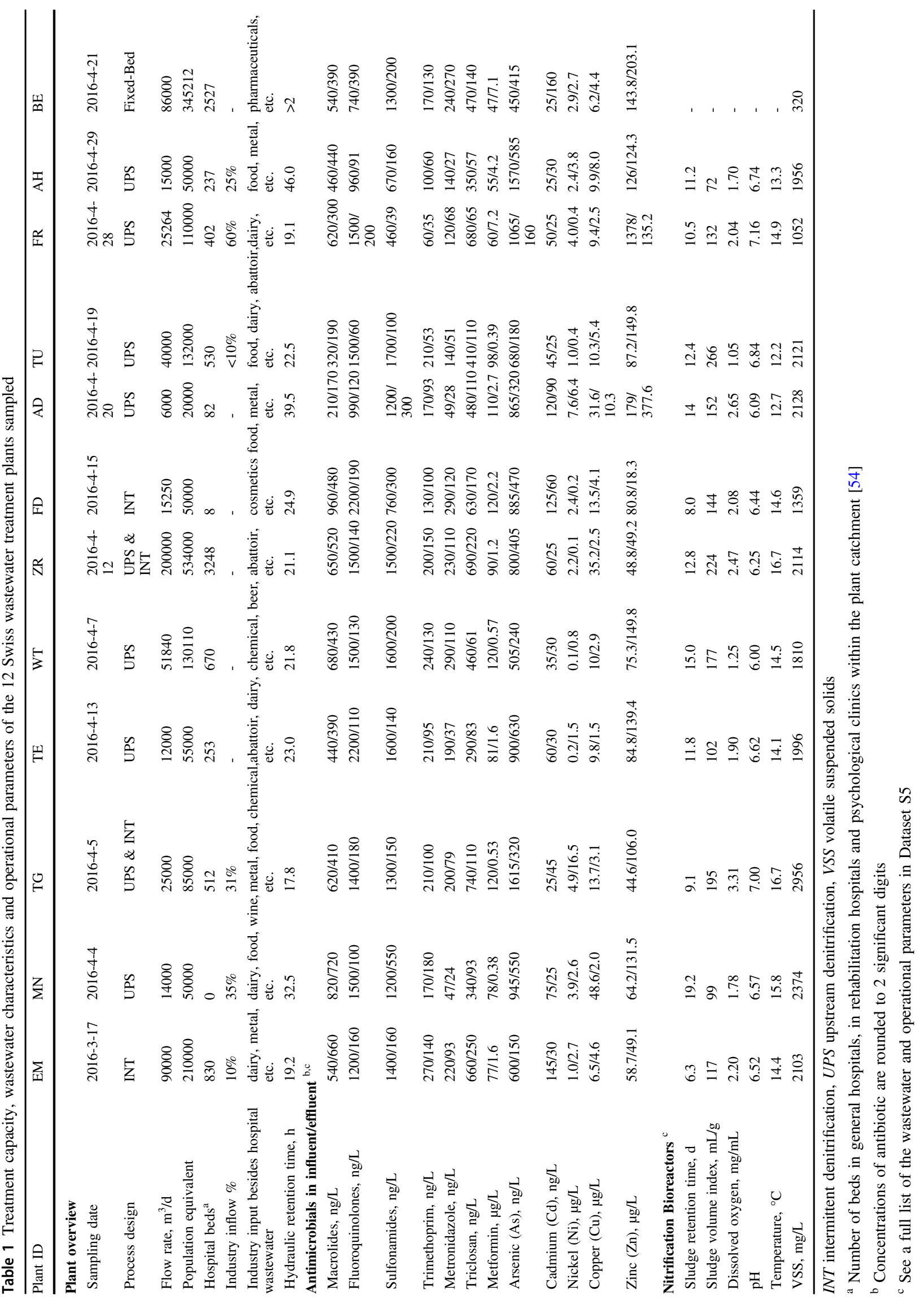


antibiotic resistance determinants through selection or coselection [21-24]. Although co-selection is well demonstrated at the levels of species and population [23], whether the sub-inhibitory wastewater antibacterial residues may lead to trackable community resistance selection remains unclear. So far, no data is available on the extent to which resistome genes are expressed in WWTPs. Studying resistance gene expression could give important hints, which, if any, of these functions are active, and whether the activity changes across compartments or in response to environmental stressors. Importantly, determining the extent to which resistance determinants (i.e., bacteria, genes and transcripts) are selected for and horizontal gene transfer is facilitated by environmental conditions within WWTPs would inform policy decisions in risk assessment and resistance surveillance for preventing dissemination of antibacterial resistance to the environment.

In this study, we used meta-omics approaches benchmarked with mRNA internal standards and qPCR analysis to build quantitative inventories of resistome genes, specifically ARG, biocide resistance genes (BRG), and metal resistance genes (MRG) in 12 communal WWTPs, providing a highly resolved view of the flow of resistance genes and their transcription in this system (Fig. 1). In the context of this manuscript, a resistome is thus understood as the collection of these resistance genes in the metagenome of a sample. By comparing the abundance and transcription levels of resistance genes across treatment compartments, we determined the factors that best predict the composition and transcription of the resistome among a wide range of biotic and abiotic (i.e., physicochemical and operational) variables. Through gene assembly and co-localization analysis, we obtained reliable ARG identification and additional information on co-located genes to predict ARGs mobility incidence (M\%) and phylogenetic distribution. The results we obtained reveal how the conventional treatment process strongly influences resistance genes and their transcriptional activities within wastewater treatment stages. Our insights provide useful guidance to the risk assessment and control strategy of WWTP discharge of resistance determinants.

\section{Materials and methods}

A full version of the Materials and methods are available in the Supplementary Information (SI).

\section{Biomass and liquid collection}

For DNA and mRNA analysis, biomass was collected from post-primary clarifier influent, denitrifying and nitrifying bioreactors, and secondary clarifier effluent of 12 Swiss
WWTPs between March and April 2016 (Table 1), as described in the SI. Filtered liquid samples were collected for in-lab chemical analysis (Supplementary Fig. S2).

\section{mRNA internal standards}

mRNA internal standards were spiked immediately after cell lysis in known copy numbers to determine volume-based or biomass-based absolute copy numbers for transcript type (i.e., copies/L or copies/g of biomass measured as volatile suspended solids). This approach circumvents the limitations of non-spiked metatranscriptomic datasets, which only provide relative abundance information [25-27]. Two mRNA standards without poly(A) tails (to mimic prokaryotic and organelle mRNAs), BMS5 and BMS6, were synthesized by plasmid linearization and in vitro transcription based on a method modified from [26], as described in the SI.

\section{RNA processing for metatranscriptomes}

Total RNAs were extracted from tube pellets and filters using the RNeasy Mini Kit (Qiagen, Germany) after cell lysis in a FastPrep instrument (MP Biomedicals) for $40 \mathrm{~s}$ (at the speed of $6.0 \mathrm{~m} / \mathrm{s}$ ) and the spiking of mRNA internal standards into the cell lysate, as described in SI. Then, the residual DNA were digested by two successive treatments with the TURBO DNA-free Kit Kit (Invitrogen, Carlsbad, CA) and mRNA was enriched from the digested total RNA samples using illumina Ribo-zero rRNA Removal Kit. cDNA libraries were generated using the rRNA-depleted RNA by NEBNext ${ }^{\circledR}$ Ultra RNA Library Prep Kit (NEB, USA) following the manufacturer's instructions.

\section{DNA processing for metagenomes}

Genomic DNA was extracted using the FastDNA ${ }^{\circledR}$ SPIN Kit for Soil (MP Biomedicals, France), following the manufacturer's instructions. The DNA extracts were then split and used for construction of metagenomic libraries, 16S rRNA gene amplification, and quantitative polymerase chain reaction (qPCR), as described below. Metagenomic libraries were generated from $1 \mu \mathrm{g}$ DNA per sample using NEBNext ${ }^{\circledR}$ Ultra $^{\mathrm{TM}}$ DNA Library Prep Kit for Illumina (NEB, USA), following manufacturer's recommendations.

\section{$16 S$ rRNA gene amplification and $q P C R$}

The V3-V4 hypervariable regions of bacterial 16S rRNA genes were amplified using genomic DNA and the forward primer $338 \mathrm{~F}$ and reverse primer $802 \mathrm{R}$ [28]. Bacterial $16 \mathrm{~S}$ rRNA gene, class 1 integron-integrase gene (intI1), and sulfonamide resistance gene sull were quantified with qPCR using LightCycler $^{\circledR} 480$ Probes Master (Roche, 
Basel, Switzerland) and Roche LightCycler ${ }^{\circledR} 480$ II. Details on the primer sets and PCR conditions used were available in the SI.

\section{Sequencing}

The constructed DNA and cDNA libraries were sequenced on the Illumina's Hiseq4000 platform using a paired-end $(2 \times 150)$ sequencing strategy at the NOVOGENE (Beijing). The 16S rRNA gene amplicons were sequenced on the Illumina's Miseq platform using a paired-end $(2 \times 250)$ sequencing strategy at the Microsynth (Switzerland).

\section{Analytical chemistry}

Dissolved antibacterial pharmaceuticals in the samples were measured by liquid chromatography triple quad mass spectrometry with electrospray ionization. Dissolved metals were measured by high-resolution inductively coupled plasma mass spectrometry. Different forms of dissolved inorganic nitrogen and phosphate were measured using SKALAR SAN ${ }^{++}$Continuous Flow Analyzer (Skalar, Breda, Netherlands). Dissolved total organic carbon was measured on a TOC-L TOC Analyzer (Shimadzu).

\section{Bioinformatics and statistics}

The bioinformatics and statistical analysis of metagenomes, metatranscriptomes, and 16S rRNA gene amplicon data are described in detail in the SI (Fig. S2). Identification of antibiotic, biocide and metal resistance genes was based on similarity search against a concatenated protein database of The NCBI Reference Sequence Database (RefSeq release 78) [30], The Comprehensive Antibiotic Resistance Database (CARD v1.0.1) [29, 31], Stuctured Antibiotic Resistance Genes Database (ARDB v1.1) [32], Antibacterial Biocide and Metal Resistance Genes Database (BacMet v1.1) [24] and functionally validated ARGs [33-35], followed by cross validation using hmmscan search against Resfams (v1.2) [36], keyword match, and manual inspection.

\section{Results}

\section{Gene inventories of WWTPs}

Gene inventories of microbiomes were built from influent, bioreactor, and effluent metagenomes of 12 communal WWTPs (Table 1). Bioinformatics analysis of 47 metagenomes (16.6 to 22.3 million reads each) allowed us to identify 9,151,591 non-redundant open-reading frames (ORFs) with contig N50 length of $1.82 \mathrm{~kb}$ (Dataset S1).
Based on protein sequence-based homology search coupled with string match and manual inspection (see methods), we predicted 16,554 ORFs as ARGs and 7465 ORFs as biocide and/or metal-resistant genes from all samples (Fig. 2a). These are carried on a total of 40,971 resistance contigs with N50 length of $18.8 \mathrm{~kb}$ (Dataset S2). From all resistance contigs, 7687 ORFs co-located with resistance genes were identified as mobility indicators (iMGE) by string match of their annotations using keywords, such as transposase, plasmid, and integrase [33].

The resistance genes were further assigned to 109 resistance "Types" by the antibacterial agents to which they were predicted to confer resistance to (Dataset S3). The most frequent ARG types were multidrug, aminoglycoside, beta-lactam, macrolide, teicoplanin, and tetracycline (Fig. 2b), representing three classic resistance mechanisms: antibiotic efflux mainly by Resistance-Nodulation-Cell Division (RND)-type, ATP-binding cassette (ABC)-type, Major facilitator superfamily (MFS)-type multidrug efflux pumps, antibiotic inactivation (e.g., beta-lactamase), and modification of antibiotic targets.

\section{Resistance genes shared by wastewater treatment compartments}

How many resistance genes traverse WWTPs and whether they are differentially expressed remains largely unknown, although answers to these questions are critical to address the roles of dispersal and local enrichment of antibacterial resistance within WWTPs. The use of a cross-sample mapping strategy enabled us to quantify numerous resistance genes that were present in a sample, but not successfully assembled from its individual metagenome (Fig. S3). Based on the mapping results, a number of quantitative metagenomic and metatranscriptomic metrics were computed and used to measure the relative and absolute abundance of microbial genes and transcripts (Table S1).

Overall, we found that while each compartment harbored unique sets of ARGs (Fig. 3a) and ARG transcripts (Fig. 3b), all compartments shared $7.4( \pm 4.1) \%$ of ARGs and $2.6( \pm 0.9) \%$ of ARG transcripts. This small core gene subset of the resistome (i.e., core resistome) was quite abundant (Fig. 3a). Similar results were found for the BRGs and MRGs (Table S2), as well as their gene transcripts (Table S3), revealing wastewater-driven dispersal of certain abundant and transcribed resistance genes or selective outgrowth of the bacteria carrying such genes throughout the WWTPs. Remarkably, $10.7 \pm(2.7) \%$ of ARGs (Fig. 3a), $9.4 \pm(2.2) \%$ of BRGs, and 10.5 $\pm(2.6) \%$ of MRGs undetectable in the influent samples became subdominant in the downstream compartments (Table S2), implicating their selective enrichment within each compartment. In contrast, 


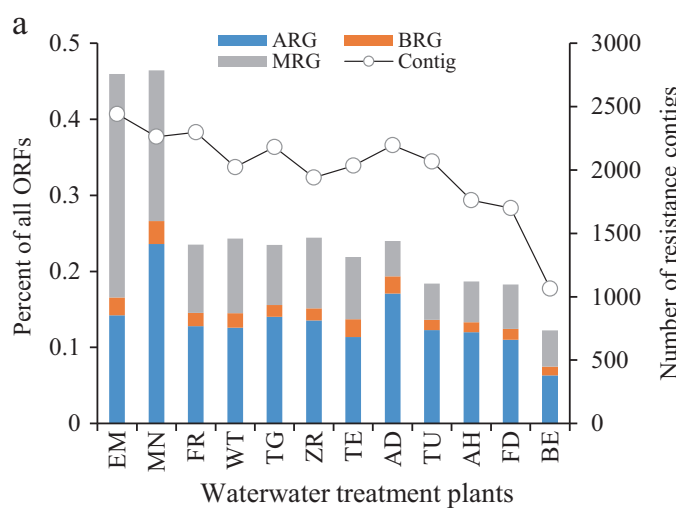

Fig. 2 Antibiotic (ARG), biocide (BRG), and metal (MRG) resistance genes predicted from influent, bioreactors, and effluent metagenomes of 12 communal wastewater treatment plants (WWTPs). a Percent of non-redundant open-reading frames (ORFs) predicted as resistance

$70.8 \%$ of the non-redundant ARGs detected in the influent samples were no longer represented in the effluent samples.

\section{Cross-compartmental differences in resistance gene transcription}

We used quantitative metaomic approaches to absolutely quantify gene abundance and transcription throughout communal WWTPs (Table S1). We demonstrated high reproducibility in transcript abundances in three metatranscriptomes spiked with mRNA internal standards $\left(R^{2}>\right.$ 0.99 , Fig. S4). Using the sulfonamide resistance gene sull as an example, we also found strong correlations between gene abundance derived from our quantitative metagenomic approach and the qPCR method (Fig. S5). To account for the significant change in the microbial biomass concentration (Table S4), bacterial 16S rRNA gene copies (Fig. S6a) and gene concentration (Fig. S6b) across WWTP compartments $(P<0.001)$, transcript copies were scaled to biomass concentration (transcript copies per-gram-of-biomass) and to gene copies of the same gene/gene type (transcript copies per gene copy) to explore differential patterns of resistance gene transcription across samples.

The absolute and relative transcript abundance metrics of the WWTP resistomes were significantly $(P<0.05)$ different across treatment compartments (Fig. 3e, f), consistent with the significant cross-compartmental variations in the relative (Fig. 3c) and absolute (Fig. S7) abundances of resistance genes. Relative to the influent and effluent, the nitrifying and denitrifying bioreactor sludge had significantly higher per-liter transcript copies of antibiotic, biocide, and metal resistance genes (Fig. 3d). The strong correlations of all resistance gene categories with biomass

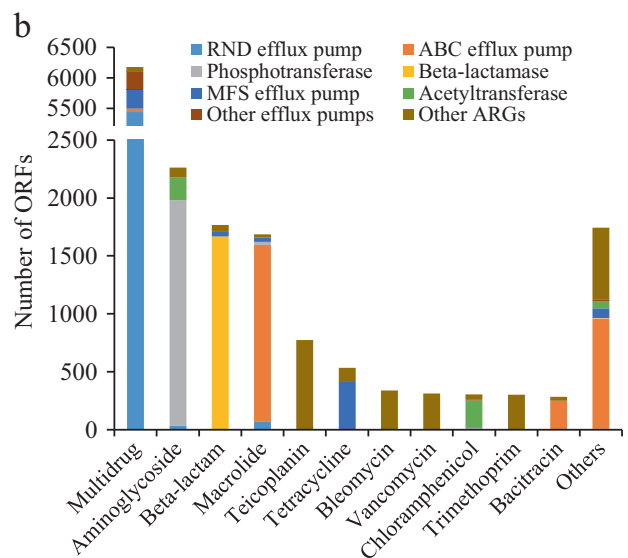

genes (left $y$-axis) and number of resistance contigs (right $y$-axis) for each WWTP (Table 1). b Number of ORFs assigned to major mechanisms for antibiotic resistance

metrics (Spearman's $r_{\mathrm{s}}>0.75$, see the network in Fig. S8) support the expectation that bacterial biomass is the main driver on the variations in the total concentration of both resistance genes and transcripts throughout the WWTPs. In contrast, the effluent and influent had significantly higher transcript copies per-gram-of-biomass (Fig. 3e) and transcript copies per gene copy (i.e., expression ratio, Fig. 3f) of resistance genes, compared with the bioreactor sludge. Notably, we observed significantly higher relative abundance for both class 1 integron-integrase gene (Fig. S6a) and resistance genes (Fig. 3c) in the effluent than in the influent. These results together suggest that conventional secondary WWTPs release bacterial populations in which resistance genes and/or class 1 integrons are significantly enriched and that express these genes.

We further checked which types of antibacterial resistance genes were upregulated and enriched in the effluent relative to the influent. Based on the relative change in the transcripts per-gram-of-biomass of the most abundant resistance types for antibiotics, biocides, and metals (Fig. 4a), we found that the transcription of most resistance types increased significantly $(* P<0.05)$ from the influent to effluent (see red bars, Fig. 4a). This pattern was most pronounced for resistance types including four antibiotic classes (tetracycline, trimethoprim, bleomycin, and polymyxin), three biocides (e.g., hydrogen peroxide), and one metal (iron). Likewise, most resistance types showed higher average transcript copies per gene copy in the effluent than the influent (Fig. 4b), suggesting that transcription of these resistance genes could be upregulated in at least a subset of all WWTPs examined. However, the lack of significant differences in the averages of transcript copies per gene copy $(P \geq 0.05)$ indicates that the increase in transcripts per- 

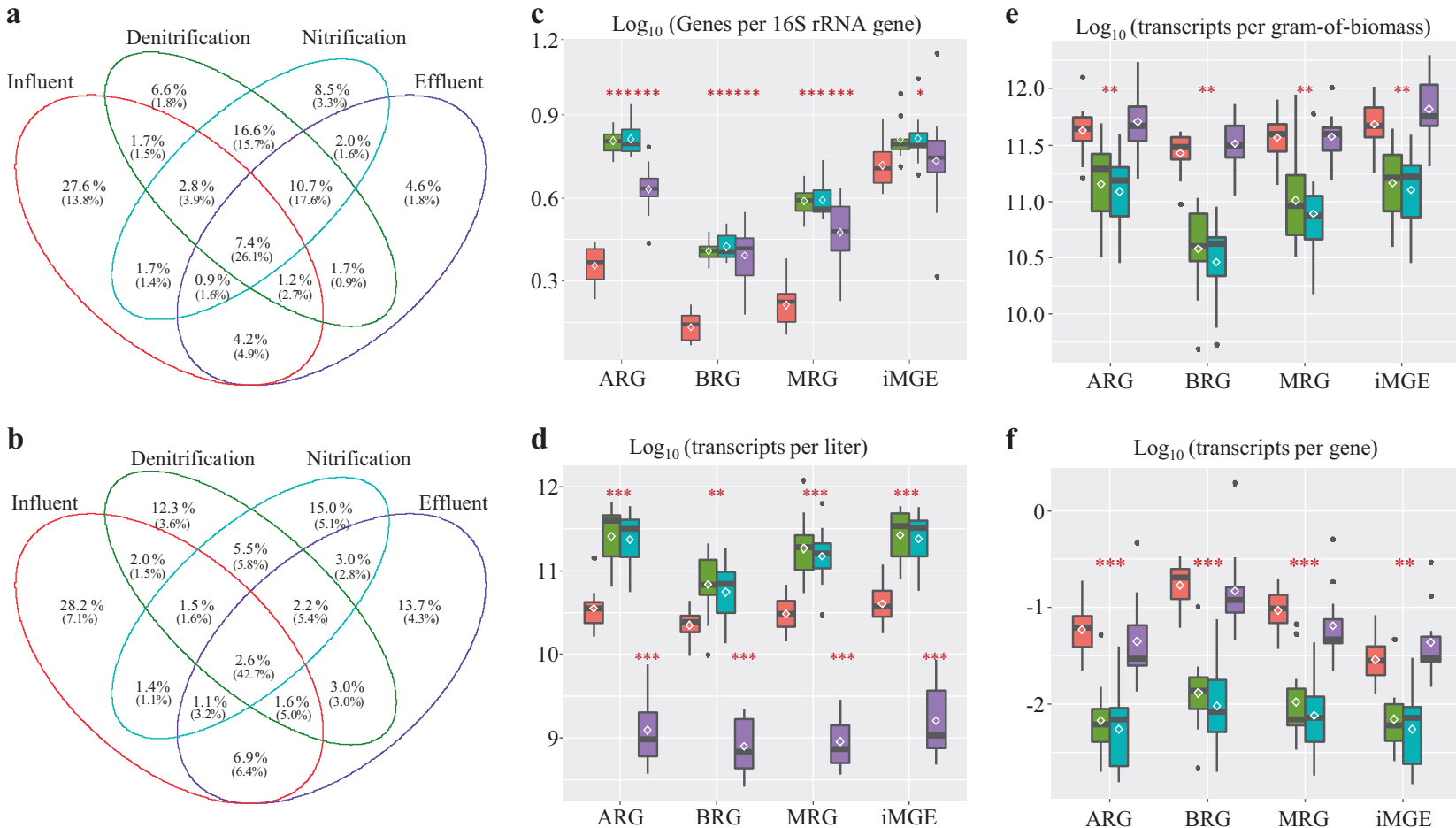

Fig. 3 Cross-compartmental variation of the richness and abundance of genes, transcripts, and mobility indicators of the WWTP resistomes. Four compartments: influent (red); denitrification (green); nitrification (cyan); effluent (purple). a, b Shared and unique percent richness (relative abundance) of ARGs (a) and ARG transcripts (b). Overall, $7.4 \%$ of ARGs and $2.6 \%$ of ARG transcripts detected in all compartments account for $26.1 \%$ of the sum for relative abundance of all ARGs (a) and $42.7 \%$ of the sum for relative abundance of all ARG transcripts (b), revealing the persistence of certain abundant resistance genes that are transcribed throughout WWTPs. c-e gene copies per 16S rRNA gene $(\mathbf{c})$, transcripts per liter $(\mathbf{d})$, transcripts per-gram-of-

gram-of-biomass largely originates with increases in the relative abundance of resistant bacteria. Indeed, the significant increase in relative abundance of most types of resistance genes, as measured by gene copies per copy of $16 \mathrm{~S}$ rRNA gene (GP16S, Fig. 4b), agrees with the significant increase of antibiotic, biocide, and metal resistance gene copies per-gram-of-biomass (Fig. S7b). These results remarkably suggest substantial relative enrichment of a broad set of antibacterial resistance genes after conventional secondary wastewater treatment.

\section{Mobility incidence and biotic and abiotic drivers of WWTP resistomes}

Co-localization or co-occurrence analysis between resistance genes and mobility indicators has been used to assess resistance mobility with regard to the potential for horizontal dissemination $[5,22,25,33]$. To quantify mobility potential of resistance genes, we define "mobility incidence" (M\%) as the percentage of resistance gene encoding biomass (e), and transcripts per gene (f). Boxes denote the interquartile range between the 25th and 75th percentiles, respectively, the line and white diamond inside denote the median and average value, black dots denote outliers and asterisks indicate significant different mean values (adjusted $P^{* * *}<0.001<* *<0.01<*<0.05$ ), compared with influent, which is checked by permutational Student's $t$-test with 10,000 simulations $(n=11)$. For any downstream compartment with significantly different means $(*)$ with influent, there is also a significant difference $(P<0.05)$ between their medians (checked by Mann-Whitney $U$-test) (color figure online)

contigs flanked with at least one co-occurring mobility indicator (iMGE) in all resistance contigs. Using resistance contigs assembled from all metagenomes, antibiotic, biocide, and metal resistance genes scored a mobility incidence of $8.6 \%, 11 \%$, and $20 \%$, respectively. We then classified all resistance genes by their mobility incidence. This innovative method enables the identification of resistance types, subtypes, or genes that tend to be more mobilized than others in any environmental resistome. We found that in the WWTPs examined "highly mobilized" (>95\% mobility) antibiotic resistance types included sulfonamide and mercury resistance, whereas "poorly mobilized" $(<5 \%$ mobility) ones included polymyxin and nitroimidazole resistance (Table S5). At the subtype level (Table S6), we found 21 highly mobilized subtypes encoding resistance functions to carbapenems (e.g., $O X A-58$ and $O X A-181$ ), oxacillin (e.g., $O X A-10)$, macrolides (ermB and $m e l)$, sulfonamide (sul1, sul2, and sul3), trimethoprim (dfrB3), copper ( $\operatorname{ctp} G)$, mercury (e.g., merE and merT), silver (silP), and etc. 
Fig. 4 The relative change of transcript and gene abundance of antibiotic, biocide, and metal resistance genes from postprimary clarifier influent to secondary effluent. Relative change is defined as the difference between effluent and influent values divided by the maximum value, thus positive (negative) values indicate increase (decrease) after wastewater treatment. a Top $x$ axis: relative change (bars) in transcript copies per-gram-ofbiomass (TPB) from influent to effluent; bottom $x$-axis: gene copies per liter of effluent (gray circles). b Relative change in transcript copies per gene copy (TPG) and gene copies per 16S rRNA gene (GP16S). The significance of mean difference in each metric between influent and effluent is tested by permutational Student's $t$-test with 10,000 simulations $\left(P^{* * * *}\right.$ $<0.001<* *<0.01<*<0.05, n$ $=11$ ). The data suggests massive increases in the expression ratio, per-grambiomass transcript copies, and relative abundance of most antibacterial resistance types (see red bars and cells). TPP tetraphenylphosphonium (color figure online) a

Increase
Decrease

Relative change in transcript copies per gram of biomass

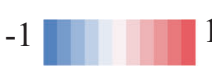

b

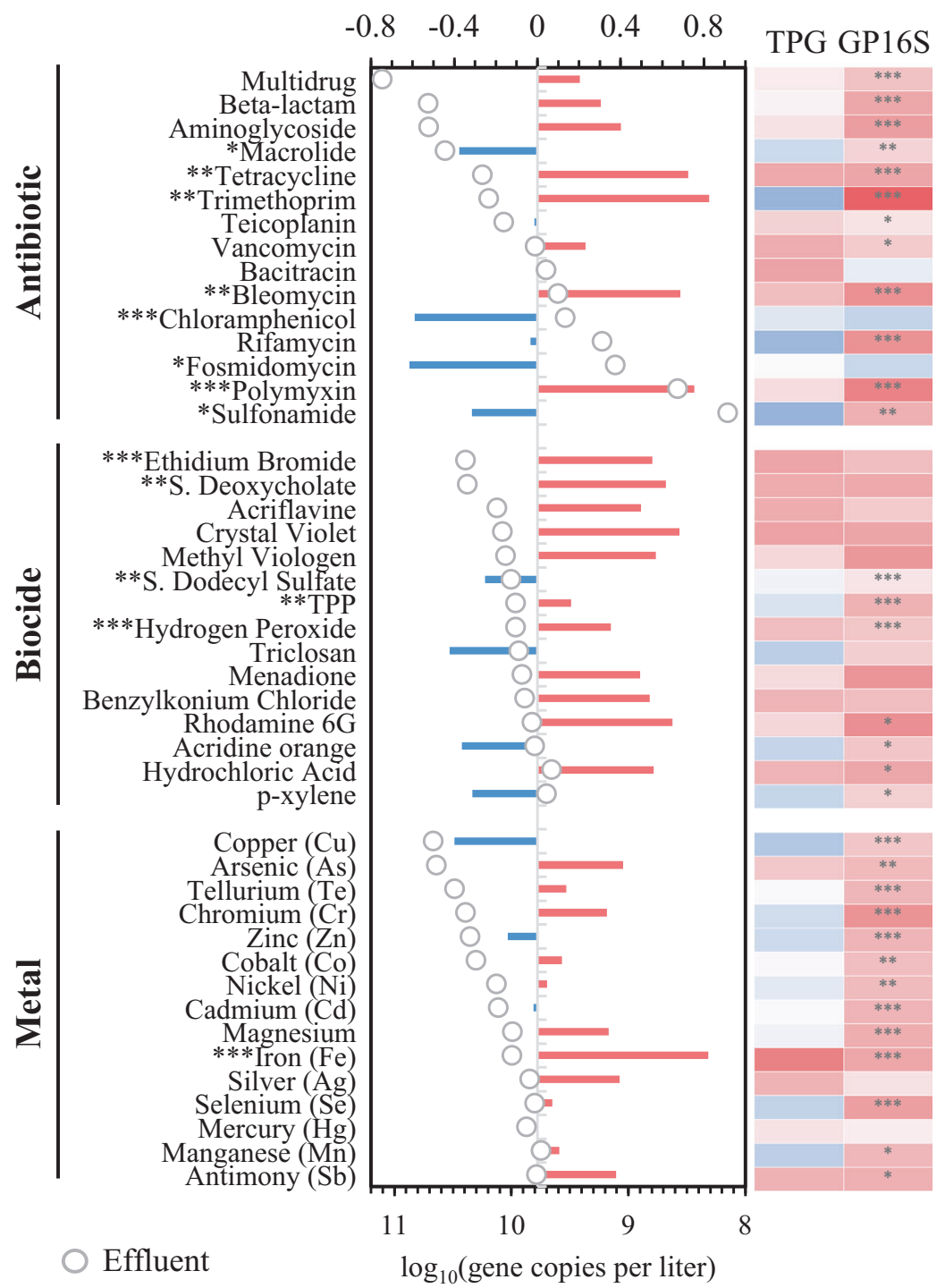

We further compared the relative strength of biotic factors (i.e., mobility elements, biodiversity, and biomass) and abiotic factors (i.e., antimicrobials, wastewater indexes, and operational parameters) in explaining the compositional variances of WWTP resistomes (Dataset S5). Redundancy analysis showed that the variances of both resistome gene and transcript compositions in the influent and effluent were best explained exclusively by biotic variables representing genetic mobility, including intI, resolvase and conjugative transfer protein, suggesting that changes happen primarily in the mobilized resistome. Bacterial alpha-diversity metrics including Shannon's H and Simpson's E (Table 2) also explained part of the variances, indicating the importance of community composition. In contrast, in the nitrifying and denitrifying bioreactors (Table S7), smaller but significant parts of resistome compositional variances were explained by three nitrogen metrics, three operational parameters, two metals (i.e., cadmium and nickel), and seven pharmaceuticals (e.g., levofloxacin, trimethoprim, and sulfamethoxazole). We also identified significant positive correlations $(P<0.05$, Table S8) between the concentration $(\mathrm{ng} / \mathrm{L})$ of measured antibiotics (i.e., macrolides, sulfonamides, lincosamide, trimethoprim, and vancomycin) and the concentration of certain ARGs (170 instances, e.g., Fig. S9a-c) or ARG transcripts (43 instances, e.g., Fig. S10a-c). The majority of these correlations were found between an antibiotic class and ARGs (Fig. S9d-h) or ARG transcripts (Fig. S10d-f) conferring resistance to a different antibiotic class, i.e., correlations that could theoretically be derived from gene co-selection or co-expression. 
Table 2 Redundancy analysis showing percent variation in the wastewater resistome composition explained by biotic and abiotic variables

\begin{tabular}{|c|c|c|c|c|c|c|c|c|c|c|c|c|}
\hline & \multicolumn{6}{|c|}{ Gene composition } & \multicolumn{6}{|c|}{ Transcript composition } \\
\hline & \multicolumn{3}{|c|}{ Influent } & \multicolumn{3}{|c|}{ Effluent } & \multicolumn{3}{|c|}{ Influent } & \multicolumn{3}{|c|}{ Effluent } \\
\hline & ARG & BRG & MRG & ARG & BRG & MRG & ARG & BRG & MRG & ARG & BRG & MRG \\
\hline \multicolumn{13}{|l|}{ Biotic variables } \\
\hline intI1/16S & 34.5 & 28.7 & 27.9 & 27.8 & 22.5 & 32.1 & 24.9 & 18.8 & 18.8 & 33.2 & 16.7 & 18.9 \\
\hline Resolvase/16S & 35.7 & 31.1 & 30.1 & 26.3 & 20.4 & 32.5 & 28.5 & 20.8 & 21.2 & 39.1 & 14.8 & 18.9 \\
\hline CTP/16S & 34.5 & 29.4 & 28.9 & 28.6 & 24.8 & 35.7 & 25.6 & 20.1 & 19.2 & 42.1 & 17.6 & 21.1 \\
\hline Shannon's $H$ & & & & 29.4 & 25.7 & 33.6 & & & & 40.3 & 17.1 & 20.6 \\
\hline Simpson's $E$ & & & & 14.2 & 14.1 & 16.1 & & & & 30.8 & & 10.4 \\
\hline \multicolumn{13}{|l|}{ Abiotic variables } \\
\hline VSS (mg/L) & 13.3 & 9.8 & 14.1 & 7.9 & 13.7 & 11.8 & & 16.3 & & 16.9 & & 10.4 \\
\hline $\begin{array}{l}\text { Nitrate nitrogen } \\
(\mathrm{mg} / \mathrm{L})\end{array}$ & & & & 10.8 & & & & & & & & \\
\hline $\begin{array}{l}\text { Total nitrogen } \\
(\mathrm{mg} / \mathrm{L})\end{array}$ & & & & 12.3 & & & & & & & & \\
\hline $\mathrm{pH}$ & 24.7 & 19.4 & & & & & & & & & & \\
\hline $\begin{array}{l}\text { Ciprofloxacin } \\
\text { (ng/L) }\end{array}$ & & & 14.5 & & & & & & 15.9 & & & \\
\hline Triclosan (ng/L) & & & & & & & & 10.3 & & & & \\
\hline
\end{tabular}

Only variables and values with significant constraints in the RDA tests $(P<0.05,1000$ permutation $)$ are shown, and a full list of the tested variables are available in Dataset S5

intI1 class 1 integron-integrase gene, 16S, 16S ribosomal RNA gene, CTP conjugal transfer protein-coding gene, VSS volatile suspended solids

\section{Interconnected WWTP resistomes and microbiomes}

Bacterial phylogeny structures soil resistomes [33]. To test if this was the case in our dataset, we used ordination to follow structural variations in the resistomes (Fig. 5 and S10) and microbiomes (Fig. S12) both between and within treatment compartments. The samples consistently clustered into three main groups by treatment compartment with bioreactor samples closely clustered together, whether the analysis was based on abundance metrics of antibiotic, biocide, and metal resistance genes (Fig. 5a-c and Fig. S11a-c) or transcripts (Fig. S11d-1). Consistent with the resistomes, the microbiomes also clustered by treatment compartment, whether a dissimilarity metric of bacterial abundance (Bray-Curtis), phylogeny (unweighted UniFrac), or both (weighted UniFrac) was used (Fig. S12). The ordinations for both resistomes and microbiomes typically showed higher within-cluster variances for the effluent samples, whereas within-cluster variances were typically smaller for the influent samples, reflecting a role of wastewater treatment in the divergence of the microbial community structure.

The structural correlations between resistome and microbiome were computed and visualized based on procrustes analyses (Fig. 5d-f). When all the treatment compartments were considered, Bray-Curtis distances calculated from abundance metrics of ARGs (d), BRGs (e) or MRGs (f) significantly $(P<0.001)$ correlated with both bacterial
OTUs ( $r=0.81-0.97$, Fig. $5 \mathrm{~d}-\mathrm{f})$ and taxa (i.e., at the genus, family, order, class, and phylum levels, Table S9) inferred from 16S rRNA sequence data, whether a dissimilarity metric of abundance (Bray-Curtis), phylogeny (unweighted UniFrac), or both (weighted UniFrac) was used. Likewise, Bray-Curtis distances calculated from transcript abundance metrics of all three categories of resistance genes also significantly correlated $(P<0.001, r=0.56-0.83)$ with both the bacterial abundance and phylogenetic structure (Table S9). On the other hand, resistome composition within treatment compartments also significantly $(P<0.05)$ correlated with abundance and/or phylogeny-based bacterial community structure (Table S10). If horizontal gene transfer occurs at very high frequencies, we might expect increasingly weaker correlations between resistome and phylogenetic structure from inflow to effluent, but this was not observed. Combined, the resistome composition correlates with both the phylogenetic (UniFrac) and taxonomic (BrayCurtis) distance metrics of community structure across and within treatment compartments, revealing a close relationship between resistome composition and bacterial phylogeny.

\section{Discussion}

The power of metagenomics and bioinformatics have been demonstrated in exploring diversity of environmental ARGs 


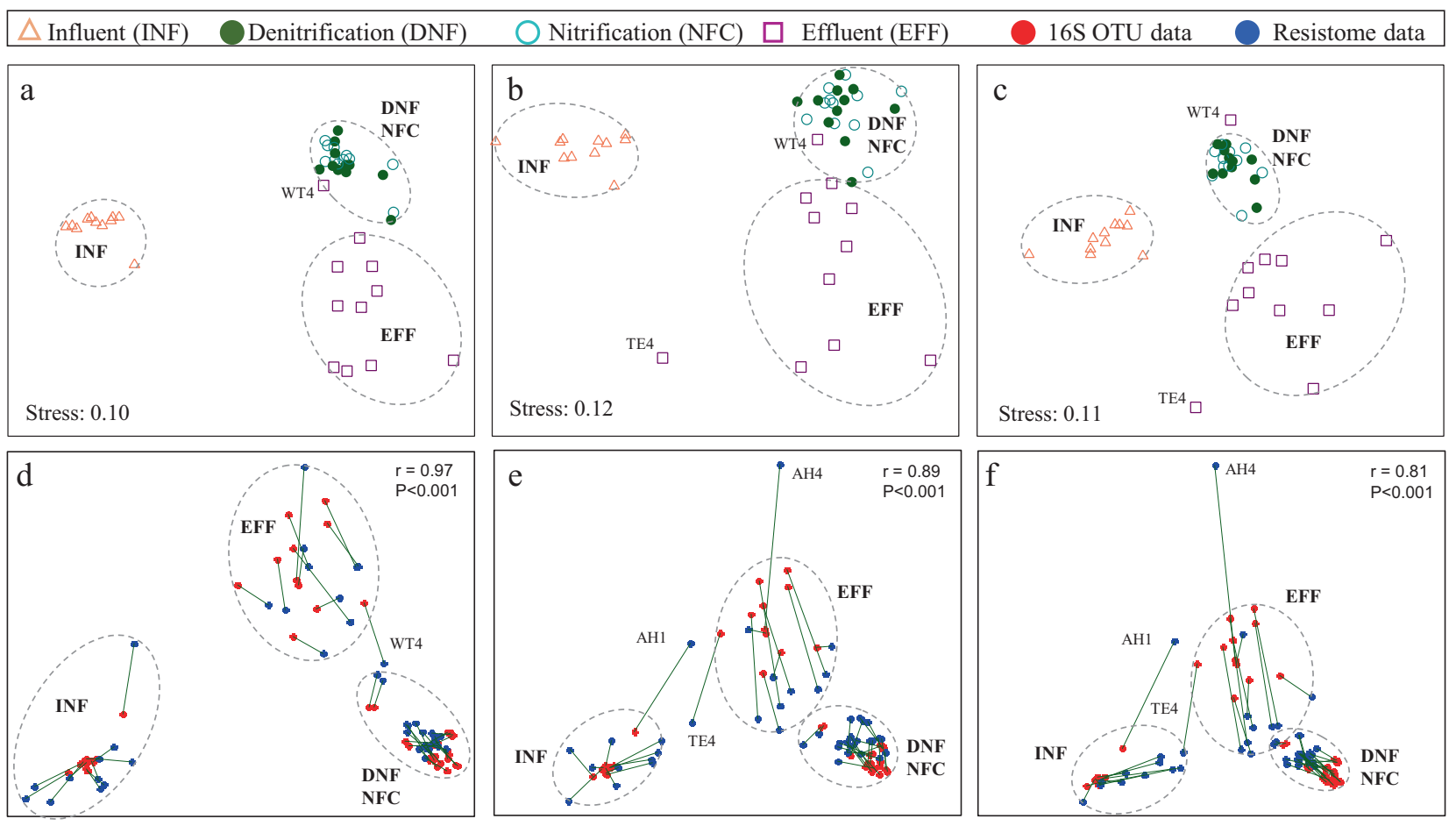

Fig. 5 Resistome composition correlates with bacterial community composition and phylogeny across wastewater treatment compartments. a-c Non-metric multidimensional scaling plots depict BrayCurtis distances between treatment compartments based on relative abundance of antibiotic (a), biocide (b), and metal (c) resistance genes in the metagenomes. d-f Procrustes analyses depict significant $(P<$
$0.001)$ and strong $(r>0.85)$ correlations between bacterial community composition (Bray-Curtis, red circles) and content of antibiotic (d), biocide (e), and metal (f) resistance genes (Bray-Curtis, blue circles), respectively. OTU operational taxonomic unit. IDs were labeled for samples outside compartment-defined sample clusters (Dataset S1) (color figure online)
$[5,13,15,37,38]$. However, the absolute quantification of a broad-spectrum of ARGs and their transcripts remains challenging. We demonstrated the integration of metaomic approaches with mRNA internal standards and qPCR data of marker genes (e.g., 16S rRNA gene) as a powerful methodology to realize both absolute and relative quantification of a broad-spectrum of microbial community genes and transcripts within a complex microbial ecosystem like WWTPs. Using these techniques, we provide extensive information on the fate and expression of the WWTP resistome genes, and influential biotic and abiotic factors.

\section{Fate and expression of antibacterial resistance genes}

Our data confirm previous findings that conventional WWTPs remove the majority of bacterial cells and with it resistance genes. Previous studies have presented contradicting evidence regarding the removal vs. enrichment of ARGs in WWTPs [6, 17, 39-42]. General conclusions remain difficult because of the discrepancies in the types of ARGs reported, abundance metrics used (i.e., relative or absolute), and/or normalization methods implemented (e.g., against $16 \mathrm{~S}$ rRNA gene or biomass). Our data strongly supports the notion that WWTP are sites for the relative enrichment of antibacterial resistance genes and class 1 integrons, as we found a surprisingly consistent increase in the relative abundance of most resistance genes and the class 1 integron-integrase gene intIl. While the relative enrichment of ARGs is also noticed in WWTPs elsewhere $[17,40,41]$, the release of class 1 integrons from wastewater systems deserves further research on their potential clinical relevance and environmental risks in the receiving environment [43].

Further, quantitative metatranscriptomics suggests that resistance genes are differentially expressed across the WWTP compartments, which provides credence to the idea that the resistance activity is influenced by environmental conditions during wastewater treatment. The constantly fluctuating physicochemical composition of influent wastewater and rapidly changing redox conditions from one treatment compartment to the next can expose microorganisms within WWTPs to rapidly varying stress. The expression of resistance genes could thus be tied to a general stress response that is not directly linked to the presence of suspected specific stressors such as measured antibiotics or metals. The impact of such specific agents is, therefore, discussed in detail below. The redox contrast between denitrification and nitrification 
compartments at least did not result in an overall differential expression of resistance genes (Fig. 3d-f). We have further demonstrated that (i) the core resistome genes are persistent, abundant, and transcribed in all the WWTP compartments and (ii) resistance genes and mobility indicators are more transcriptionally active in the secondary effluent than in activated sludge bioreactors. These findings indicate that some resistance genes and resistant bacteria are highly recalcitrant to conventional secondary treatment processes and that these facilities release abundant actively transcribed resistance genes together with mobile genetic elements into the receiving environment. It should be noted, that the expression ratios of ARGs in WWTPs we detected are far lower than one transcript per gene copy. While these values are comparable to those reported with the same methodological approach for biogeochemically relevant genes in the Amazon River Plume [28], such values lie far below what is typically observed in organism-based studies. Further research will be needed to understand these seemingly low transcriptional activities.

\section{Biotic and abiotic drivers of the WWTP resistomes}

The relative roles of biotic and abiotic factors in shaping environmental resistome and facilitating resistance selection are poorly understood $[2,23]$. We demonstrate that biotic factors including mobility elements (intIl, conjugal transfer protein, and resolvase) and biomass play an important role in shaping compositional variations of the influent and effluent resistomes. Class 1 integrons are central players in resistance dissemination [43, 44], whose activation upon conjugative plasmid transfer allows host bacteria to rapidly develop antibiotic resistance [45, 46]. Plasmid-mediated antibiotic and metal resistance has been reported in wastewater $[6,12,14,47]$. The proportions of ARGs (5.4\%) and MRGs $(8.1 \%)$ in total plasmid-borne genes we identified are comparable to the levels in two other Swiss WWTPs (ARG: 2.5 and 4.0\%; MRG: 4.5 and 12.5\%) [14]. The strong explanatory power of mobility indicators thus shows the importance of mobilized resistance in the wastewater, and supports the use of, e.g., intIl as a general indicator of resistance [2, 43]. However, in the activated sludge abiotic factors (i.e., inorganic nitrogen, $\mathrm{pH}$, dissolved oxygen, and several antimicrobials) appear to play an additional role in shaping resistomes (Table S7). In this compartment, nutrients and oxygen are substantially consumed by activated sludge biomass and may thus act as driving forces for both community and resistome composition.

\section{Positive correlations between antibiotics and resistance}

Positive correlations were found between certain antibiotics in wastewater and "their" resistance genes and resistance gene transcripts, as well as with resistance genes conferring resistance to a different antibiotic class. On the one hand, such positive relationships, for example, those between wastewater concentrations of macrolide antibiotics, clarithromycin (76-460 ng/L) and azithromycin (46-310 ng/L) and the concentration of macrolide resistance gene $\mathrm{macB}$ (Fig. S9a-b), could be the consequence of enrichment of the resistance genes in the population based on selective pressures exerted by the antibiotic. Considering the demonstrated selection of resistant strains at very low and subinhibitory antibiotic concentration [48] this is a reasonable expectation. However, further antibiotic susceptibility tests on wastewater isolates or experimental validation with wastewater communities are required to validate this correlation-based speculation. On the other hand, the demonstrated positive correlations between wastewater antibiotics and resistance genes or transcripts of another antibiotic class could reflect co-selection of multi-resistance on the same genetic elements, i.e., co-resistance [23]. The most striking examples are the strong positive correlations found between macrolide antibiotics and both vancomycin resistance genes (Fig. S9d-e) and their transcripts (Fig. S10d-e). The co-localization or adjacency of vancomycin and macrolide resistance genes on the same genomic fragments (12 instances, Dataset S2), as well as the strong positive correlations between their absolute copies in all the four WWTP compartments $\left(R^{2}=0.77-0.93\right.$, Fig. S9f-i), are strong evidence for their co-selection, which also makes the induced expression of the vancomycin resistance genes by clarithromycin plausible (Fig. S10d-e). Another intriguing example is the strong positive relation found between the concentrations of sulfamethoxazole and transcripts of trimethoprim resistance gene $d f r B 3\left(R^{2}=0.94\right.$, Fig. S10f). A general practice of combined use of trimethoprim and sulfamethoxazole in clinical settings may facilitate their coselection. However, the observed correlations could also be inherited from selective processes in human gut bacteria of patients under treatment rather than within the WWTPs.

The above findings highlight the multi-dimensionality and complexity of environmental (co-)selection of antibiotic resistance and thus explain the inability of previous studies to assign or relate certain antibiotics to the occurrence and/or abundance of their respective resistance genes [10, 37, 4951]. In particular for the WWTP environment, one may argue that metal contaminants may also co-select for ARGs and MRGs, thus decoupling simple ' antibiotic-ARG', relationships [23]. However, our contig data do not support that such co-selection is common in the WWTPs, considering a very low incidence $(0.6 \%)$ of finding an ARG and an MRG encoded on the same resistance contig (Dataset S2). This lack of co-occurrence scenarios between MRGs and ARGs agrees with their rare co-occurrence on plasmids from natural environments $(<0.7 \%)$ [25]. However our resistance contig- 
based analysis (N50 length of $18.8 \mathrm{~kb}$ ) likely underestimates the co-selection potential if these genes are distanced on different genomic islands or multi-resistance plasmids [23].

\section{Gene mobility potentials of the WWTPs resistomes}

The average mobility incidences (M\%) of ARGs found in our WWTPs influent (10\%), activated sludge (7.1-7.8\%), and effluent $(9.8 \%)$ resistomes were comparable to those found in the human gut resistomes (14\% of 161 contigs), where horizontal gene transfer (HGT) is implicated in facilitating resistance acquisition by human pathogens [34]. In contrast, lower mobility incidence of ARGs have been reported for soils ( $0.8 \%$ of 4655 contigs [5]), where HGT is suggested to play a limited role in resistance dissemination. Remarkably, we find that of the 17,486 resistance genes shared by bacteria, the majority $(93.5 \%)$ are encoded on multiple resistance contigs (2 to 46) with considerably diverging flanking regions (Dataset S6). This novel finding of a large-scale distribution of identical resistance genes on divergent contigs derived from DNA samples from different WWTPs/compartments strongly implicates a history of substantial exchange of antibacterial resistance genes.

Moreover, three further lines of evidence suggested HGT may play a more important role in the secondary clarifiers than previously appreciated: (i) an important role of intIl, resolvase and conjugal transfer protein in structuring resistomes in the low-biomass clarified effluent rather than the thick activated sludge (Fig. S13), (ii) the higher per-gramof-biomass and per gene transcriptional activities of resistance and mobility-related genes (Fig. 3e, f), as well as higher relative abundance of intII (Fig. S6a) and ARGs (Fig. 3c) in the secondary effluent than activated sludge, and (iii) high incidences of integrases $(31 \%)$ and conjugal transfer proteins $(35 \%)$ co-located with plasmid proteins on the same resistance contigs (Dataset S2). Based on these findings, we hypothesize that contrary to our original expectations, secondary clarifier suspended bacteria, which are mostly planktonic, are exposed to higher overall stress from contaminants (e.g., per-gram-of-biomass antibiotic/ metal loadings). This results in both, stronger selection and more active transcription, of resistance-related genes compared with bacterial cells harbored within the protective activated sludge flocs. In flocs antibacterial resistance or detoxification can be achieved through extracellular inactivation (e.g., beta-lactam and aminoglycoside), exopolysaccharide binding (e.g., some metals and chemical toxins) and/or biodegradation or biotransformation (e.g., biodegradable pharmaceuticals).

Despite the evidence for gene mobilization within the resistomes of WWTP effluent, we have demonstrated that the resistome composition overall correlates tightly with the bacterial community phylogenetic and taxonomic composition (Fig. 5), suggesting that the changes to the species composition resulting from the wastewater treatment process strongly determine the effluent resistomes. While this finding agrees with a close connection found between antibiotic resistome and bacterial phylogeny in soils and human guts [33, 37], it may also reflect the existence of certain phylogenetic constraints for the horizontal dissemination of antibiotic resistance between bacterial populations.

\section{Implications for risk assessment and management of resistomes}

Our data strengthens the case for using intIl gene abundance and concentration as a general indicator of anthropogenic impacts [43], as we could demonstrate their predictive power for WWTP resistomes and relative enrichment after wastewater treatment, in accordance with the significant increase in relative abundance of resistance genes. Using mobility incidence (M\%), we are able to predict and compare the transferable potentials of resistance genes at the levels of resistance type, subtype and ecosystem, which is an important aspect for risk ranking in resistomes [52]. While the keyword-based approach used in the assessment of M\% is likely to have shortcomings-for example, we only test for co-localization of an appropriately annotated genetic element but do not confirm its function or if it actually confers mobility to the ARG-it proved a useful tool and provided believable rankings. Typical examples of $100 \%$ mobilized ARGs we identified from WWTP effluent include well-known acquired resistance genes such as $C T X-M, O X A$, and TEM family extendedspectrum beta-lactamases (ESBL) and $O X A$ family carbapenemases. Our approach could be further improved, for example, by using a verified reference database of mobility indicators instead of keywords. Besides gene mobility, risk ranking in resistomes should also consider the host pathogenicity and clinical importance of ARGs with regard to disease control in humans and/or domestic livestock [52]. We demonstrate that contig-based analysis of metagenomes can again provide a basis for such assessments: For instance, we found 11 non-redundant ARGs representing a total of 138 ORFs with $100 \%$ identity to reference sequences from known clinical isolates of human pathogens (Table 3). The high occurrence frequency of these "pathogenic" ARGs (11/12) and their transcripts (9/12) in the effluent of examined WWTPs (e.g., sull, ermB, ANT3, and $c m l A$ ) suggests that further investigation of their fate and health risk in the receiving environment is warranted.

Additional preventative or control measures of antibiotic resistance determinants in WWTP effluents may currently 
Table 3 Non-redundant antibiotic resistance genes with $100 \%$ identity to known human bacterial pathogens

\begin{tabular}{|c|c|c|c|c|c|c|c|c|c|}
\hline \multirow[t]{2}{*}{ Gene ID } & \multirow[t]{2}{*}{ Length (aa) } & \multicolumn{2}{|l|}{ Resistance } & \multirow{2}{*}{$\begin{array}{l}\text { Number of } \\
\text { sequences }\end{array}$} & \multirow{2}{*}{$\begin{array}{l}\text { Example of pathogen } \\
\text { (NCBI taxon ID) }\end{array}$} & \multicolumn{2}{|l|}{ Gene } & \multicolumn{2}{|c|}{ Transcript } \\
\hline & & Type & Subtype & & & Influent & Effluent & Influent & Effluent \\
\hline W56_28340_1 ${ }^{\mathrm{a}, \mathrm{b}, \mathrm{c}}$ & 260 & Aminoglycoside & ANT3 & 3 & A. baumannii (509173) & 12 & 5 & 10 & 8 \\
\hline W54_36555_1 $1^{\mathrm{a}, \mathrm{b}, \mathrm{c}}$ & 265 & Aminoglycoside & $A P H\left(3^{\prime}\right)$ & 9 & S. epidermidis (176279) & 12 & 3 & 7 & 2 \\
\hline W54_1320_4 $4^{\mathrm{a}, \mathrm{c}}$ & 144 & Aminoglycoside & sat-1 & 8 & B. vulgatus (435590) & 12 & 5 & 2 & 0 \\
\hline W56_17638_3 $3^{\mathrm{a}, \mathrm{b}, \mathrm{c}}$ & 278 & Aminoglycoside & $s t r B$ & 7 & K. pneumoniae (272620) & 12 & 8 & 8 & 6 \\
\hline W54_14042_1 $1^{\mathrm{a}, \mathrm{c}}$ & 281 & Beta-lactam & $O X A-58$ & 5 & A. baumannii (405416) & 11 & 4 & 7 & 1 \\
\hline W56_739_2 $2^{\mathrm{a}}$ & 425 & Beta-lactam & ampG & 6 & B. vulgatus (435590) & 12 & 7 & 0 & 0 \\
\hline W70_15043_2 $2^{\mathrm{a}, \mathrm{b}, \mathrm{c}}$ & 420 & Chloramphenicol & cmlA & 5 & K. pneumoniae (272620) & 12 & 2 & 9 & 5 \\
\hline W60_5396_4, ${ }^{\mathrm{a}, \mathrm{c}}$ & 250 & Macrolide & ermB & 15 & E. faecalis (226185) & 12 & 11 & 12 & 12 \\
\hline W54_264_15 ${ }^{\mathrm{a}}$ & 377 & Multidrug & $\operatorname{mex} E$ & 12 & B. vulgatus (435590) & 12 & 12 & 1 & 0 \\
\hline W71_4945_1 $1^{\mathrm{a}, \mathrm{b}, \mathrm{c}}$ & 309 & Sulfonamide & sul1 & 47 & S. enterica (423368) & 12 & 12 & 12 & 12 \\
\hline W56_1220_2 & 658 & Tetracycline & tet $Q$ & 19 & B. fragilis (295405) & 12 & 12 & 9 & 4 \\
\hline W72_76188_2 $2^{\mathrm{a}, \mathrm{c}}$ & 104 & Trimethoprim & dfrA14 & 2 & B. hermsii (314723) & 9 & 0 & 2 & 1 \\
\hline
\end{tabular}

The last four columns show the number of WWTPs in which the resistance gene and its transcripts are detected in the influent or effluent compartment

${ }^{a}$ Found in human bacterial pathogens, but its resistance contigs showing $<95 \%$ global nucleotide identity (at least $5 \%$ divergence) to the pathogen sequences

${ }^{\mathrm{b}}$ Found on both genomes and plasmids of at least one pathogen

${ }^{\mathrm{c}}$ Co-located with indicators of mobile genetic elements on the same resistance contig

not be a priority, unless direct health risks for humans are verified. Nonetheless, our data underscores that the absolute amounts of resistance bacteria and genes discharged by WWTPs into the environment is heavily dependent on the bacterial biomass remaining in the final effluent. Thus, any measure that substantially reduces bacterial biomass in the discharged effluent, such as an increase of sludge settleability in the secondary clarifiers [53] or membrane filtration would reduce WWTP discharge of resistance genes. In agreement with this idea, membrane bioreactors are implicated to show much higher absolute removal efficiency of some ARGs and bacteria than conventional WWTPs [18].

Acknowledgements This research was funded by Eawag Discretionary Funds (grant number: 5221.00492.009.06 DF15 / GeneFlow). We appreciate the help of MA Moran and her team with quantitative transcriptomics. We appreciate the help of P Kathriner, D Kistler, A Minder, N Zemp, and JC Walser, and thank the Genetic Diversity Center (GDC) at the ETH Zürich for providing computational resources and support. F Ju and $\mathrm{H}$ Bürgmann received financial support from the NFP72 project 407240_167116 "Swiss River Resistome". We acknowledge valuable support by PIRE program 1545756 "Halting Environmental Antimicrobial Resistance Dissemination (HEARD)".

Author contributions $\mathrm{HB}, \mathrm{FJ}, \mathrm{CM}$, and DJ designed research. FJ and $\mathrm{KB}$ performed sample collection and molecular experiments. FJ analyzed all the sequence data. TZ and XLY assisted with the classification of antibiotic resistance genes. AM, CM and $\mathrm{SH}$ conducted analytical chemistry of pharmaceuticals. FJ and $\mathrm{HB}$ wrote the manuscript. All authors contributed to critical discussion and revisions of the manuscript.
Data Availability The sequences reported in this paper have been deposited in the Metagenomics Analysis Server (MR-RAST) with project ids of mgp83169, mgp19765, mgp19780, mgp19899, mgp19773, mgp19991, and mgp21278 (see Dataset S1 and S4 for all sample ids).

\section{Compliance with ethical standards}

Competing interests The authors declare no competing interests.

\section{References}

1. Allen HK, Donato J, Wang HH, Cloud-Hansen KA, Davies J, Handelsman J. Call of the wild: antibiotic resistance genes in natural environments. Nat Rev Microbiol. 2010;8:251-9.

2. Berendonk TU, Manaia CM, Merlin C, Fatta-Kassinos D, Cytryn E, Walsh F, et al. Tackling antibiotic resistance: the environmental framework. Nat Rev Microbiol. 2015;13:310-17.

3. Vikesland PJ, Pruden A, Alvarez PJJ, Aga DS, Buergmann H, Li $X$ et al (2017). Towards a comprehensive strategy to mitigate dissemination of environmental sources of antibiotic resistance. Environmental Science and Technology 51:13061-69.

4. Bürgmann $H$, Frigon D, H Gaze W, M Manaia C, Pruden A, Singer AC et al (2018). Water and sanitation: an essential battlefront in the war on antimicrobial resistance. FEMS Microbiol Ecol 94: fiy101-fiy101.

5. Forsberg KJ, Reyes A, Wang B, Selleck EM, Sommer MO, Dantas G. The shared antibiotic resistome of soil bacteria and human pathogens. Science. 2012;337:1107-11.

6. Szczepanowski R, Linke B, Krahn I, Gartemann K-H, Gützkow T, Eichler W, et al. Detection of 140 clinically relevant antibioticresistance genes in the plasmid metagenome of wastewater treatment plant bacteria showing reduced susceptibility to selected antibiotics. Microbiology. 2009;155:2306-19. 
7. Zurfluh K, Hächler H, Nüesch-Inderbinen M, Stephan R. Characteristics of extended-spectrum $\beta$-lactamase-and carbapenemaseproducing Enterobacteriaceae isolates from rivers and lakes in Switzerland. Appl Environ Microbiol. 2013;79:3021-6.

8. Michael I, Rizzo L, McArdell C, Manaia C, Merlin C, Schwartz T, et al. Urban wastewater treatment plants as hotspots for the release of antibiotics in the environment: a review. Water Res. 2013;47:957-95.

9. Bollmann UE, Tang C, Eriksson E, Jönsson K, Vollertsen J, Bester K. Biocides in urban wastewater treatment plant influent at dry and wet weather: concentrations, mass flows and possible sources. Water Res. 2014;60:64-74.

10. Novo A, André S, Viana P, Nunes OC, Manaia CM. Antibiotic resistance, antimicrobial residues and bacterial community composition in urban wastewater. Water Res. 2013;47: 1875-87.

11. Ju F, Li B, Ma L, Wang Y, Huang D, Zhang T. Antibiotic resistance genes and human bacterial pathogens: co-occurrence, removal, and enrichment in municipal sewage sludge digesters. Water Res. 2016;91:1-10.

12. Li A-D, Li L-G, Zhang T. Exploring antibiotic resistance genes and metal resistance genes in plasmid metagenomes from wastewater treatment plants. Front. Microbiol. 2015a;6. https://www. frontiersin.org/articles/10.3389/fmicb.2015.01025/full.

13. Li B, Yang Y, Ma L, Ju F, Guo F, Tiedje JM, et al. Metagenomic and network analysis reveal wide distribution and co-occurrence of environmental antibiotic resistance genes. ISME J. 2015b;9:2490-502.

14. Sentchilo V, Mayer AP, Guy L, Miyazaki R, Tringe SG, Barry K, et al. Community-wide plasmid gene mobilization and selection. ISME J. 2013;7:1173.

15. Yang Y, Li B, Ju F, Zhang T. Exploring variation of antibiotic resistance genes in activated sludge over a four-year period through a metagenomic approach. Environ Sci Technol. 2013;47:10197-205.

16. Czekalski N, Díez EG, Bürgmann H. Wastewater as a point source of antibiotic-resistance genes in the sediment of a freshwater lake. ISME J. 2014;8:1381-90.

17. Di Cesare A, Eckert EM, D’Urso S, Bertoni R, Gillan DC, Wattiez $\mathrm{R}$, et al. Co-occurrence of integrase 1 , antibiotic and heavy metal resistance genes in municipal wastewater treatment plants. Water Res. 2016;94:208-14.

18. Munir M, Wong K, Xagoraraki I. Release of antibiotic resistant bacteria and genes in the effluent and biosolids of five wastewater utilities in Michigan. Water Res. 2011;45:681-93.

19. Luo Y, Yang F, Mathieu J, Mao D, Wang Q, Alvarez P. Proliferation of multidrug-resistant New Delhi metallo- $\beta$-lactamase genes in municipal wastewater treatment plants in northern China. Environ Sci \& Technol Lett. 2013;1:26-30.

20. Schwartz T, Kohnen W, Jansen B, Obst U. Detection of antibioticresistant bacteria and their resistance genes in wastewater, surface water, and drinking water biofilms. FEMS Microbiol Ecol. 2003;43:325-35.

21. Li L-G, Xia Y, Zhang T. Co-occurrence of antibiotic and metal resistance genes revealed in complete genome collection. ISME J. 2017;11:651.

22. Baker-Austin C, Wright MS, Stepanauskas R, McArthur J. Coselection of antibiotic and metal resistance. Trends Microbiol. 2006;14:176-82.

23. Pal C, Bengtsson-Palme J, Rensing C, Kristiansson E, Larsson DJ. BacMet: antibacterial biocide and metal resistance genes database. Nucleic Acids Res. 2014;42:D737-43.

24. Pal C, Bengtsson-Palme J, Kristiansson E, Larsson DJ. Cooccurrence of resistance genes to antibiotics, biocides and metals reveals novel insights into their co-selection potential. BMC Genom. 2015;16:964.
25. Gifford SM, Sharma S, Rinta-Kanto JM, Moran MA. Quantitative analysis of a deeply sequenced marine microbial metatranscriptome. ISME J. 2011;5:461-72.

26. Satinsky BM, Gifford SM, Crump BC, Moran MA. Use of internal standards for quantitative metatranscriptome and metagenome analysis. Methods Enzymol. 2012;531:237-50.

27. Satinsky BM, Crump BC, Smith CB, Sharma S, Zielinski BL, Doherty $\mathrm{M}$, et al. Microspatial gene expression patterns in the Amazon River Plume. Proc. Natl Acad. Sci. 2014;111: 11085-90.

28. Klindworth A, Pruesse E, Schweer T, Peplies J, Quast C, Horn M, et al. Evaluation of general 16S ribosomal RNA gene PCR primers for classical and next-generation sequencing-based diversity studies. Nucl Acids Res. 2013;41:e1. https://academic.oup.com/ nar/article/41/1/e1/1164457.

29. Yang Y., Jiang XT, Cai BL, Ma LP, Li B, Zhang AN, Cole JR, Tiedje JM* and Zhang T*. (2016). ARGs-OAP: Online Analysis Pipeline for Anti-biotic Resistance Genes Detection from Metagenomic Data Using an Integrated Structured ARG-database. Bioinformatics. 2016;32:2346-51.

30. Pruitt KD, Tatusova T, Maglott DR. NCBI reference sequences (RefSeq): a curated non-redundant sequence database of genomes, transcripts and proteins. Nucleic Acids Res. 2007;35:D61-5.

31. McArthur AG, Waglechner N, Nizam F, Yan A, Azad MA, Baylay AJ, et al. The comprehensive antibiotic resistance database. Antimicrob Agents Chemother. 2013;57:3348-57.

32. Liu B, Pop M. ARDB-antibiotic resistance genes database. Nucl Acids Res. 2009;37:D443-7.

33. Forsberg KJ, Patel S, Gibson MK, Lauber CL, Knight R, Fierer N, et al. Bacterial phylogeny structures soil resistomes across habitats. Nature. 2014;509:612.

34. Sommer MO, Dantas G, Church GM. Functional characterization of the antibiotic resistance reservoir in the human microflora. Science. 2009;325:1128-31.

35. Cheng G, Hu Y, Yin Y, Yang X, Xiang C, Wang B, et al. Functional screening of antibiotic resistance genes from human gut microbiota reveals a novel gene fusion. FEMS Microbiol Lett. 2012;336:11-16.

36. Gibson MK, Forsberg KJ, Dantas G. Improved annotation of antibiotic resistance determinants reveals microbial resistomes cluster by ecology. ISME J. 2015;9:207-16.

37. Pehrsson EC, Tsukayama P, Patel S, Mejía-Bautista M, Sosa-Soto $\mathrm{G}$, Navarrete $\mathrm{KM}$, et al. Interconnected microbiomes and resistomes in low-income human habitats. Nature. 2016;533: 212-6.

38. Zhu Y-G, Johnson TA, Su J-Q, Qiao M, Guo G-X, Stedtfeld RD, et al. Diverse and abundant antibiotic resistance genes in Chinese swine farms. Proc Natl Acad Sci. 2013;110: 3435-40.

39. Karkman A, Johnson TA, Lyra C, Stedtfeld RD, Tamminen M, Tiedje JM, et al. High-throughput quantification of antibiotic resistance genes from an urban wastewater treatment plant. FEMS Microbiol Ecol. 2016;92. https://academic.oup.com/femsec/a rticle/92/3/fiw014/2470099.

40. Mao D, Yu S, Rysz M, Luo Y, Yang F, Li F, et al. Prevalence and proliferation of antibiotic resistance genes in two municipal wastewater treatment plants. Water Res. 2015;85:458-66.

41. Bengtsson-Palme J, Hammarén R, Pal C, Östman M, Björlenius $\mathrm{B}$, Flach C-F, et al. Elucidating selection processes for antibiotic resistance in sewage treatment plants using metagenomics. Sci Total Environ. 2016;572:697-712.

42. Yang Y, Li B, Zou S, Fang HH, Zhang T. Fate of antibiotic resistance genes in sewage treatment plant revealed by metagenomic approach. Water Res. 2014;62:97-106.

43. Gillings MR, Gaze WH, Pruden A, Smalla K, Tiedje JM, Zhu YG. Using the class 1 integron-integrase gene as a proxy for anthropogenic pollution. ISME J. 2015;9:1269-79. 
44. Gillings M, Boucher Y, Labbate M, Holmes A, Krishnan S, Holley M, et al. The evolution of class 1 integrons and the rise of antibiotic resistance. J Bacteriol. 2008;190:5095-100.

45. Baharoglu Z, Bikard D, Mazel D. Conjugative DNA transfer induces the bacterial SOS response and promotes antibiotic resistance development through integron activation. PLoS Genet. 2010;6:e1001165.

46. Lacotte Y, Ploy M-C, Raherison S. Class 1 integrons are lowcost structures in Escherichia coli. The ISME Journal. 2017;7:1535-44.

47. Schlüter A, Szczepanowski R, Pühler A, Top EM. Genomics of IncP-1 antibiotic resistance plasmids isolated from wastewater treatment plants provides evidence for a widely accessible drug resistance gene pool. FEMS Microbiol Rev. 2007;31:449-77.

48. Gullberg E, Cao S, Berg OG, Ilbäck C, Sandegren L, Hughes D, et al. Selection of resistant bacteria at very low antibiotic concentrations. PLoS Pathog. 2011;7:e1002158.

49. Graham DW, Olivares-Rieumont S, Knapp CW, Lima L, Werner D, Bowen E. Antibiotic resistance gene abundances associated with waste discharges to the Almendares River near Havana, Cuba. Environ Sci Technol. 2010;45:418-24.

50. Looft T, Johnson TA, Allen HK, Bayles DO, Alt DP, Stedtfeld $\mathrm{RD}$, et al. In-feed antibiotic effects on the swine intestinal microbiome. Proc Natl Acad Sci. 2012;109:1691-6.

51. Oberlé K, Capdeville M-J, Berthe T, Budzinski Hln, Petit F. Evidence for a complex relationship between antibiotics and antibiotic-resistant Escherichia coli: from medical center patients to a receiving environment. Environ Sci Technol. 2012;46:1859-68.

52. Martínez JL, Coque TM, Baquero F. What is a resistance gene? Ranking risk in resistomes. Nat Rev Microbiol. 2015;13:116.

53. Novo A, Manaia CM. Factors influencing antibiotic resistance burden in municipal wastewater treatment plants. Appl Microbiol Biotechnol. 2010;87:1157-66.

54. Kuroda K, Itten R, Kovalova L, Ort C, Weissbrodt D, McArdell C.S. Hospital-use pharmaceuticals in Swiss waters modeled at high spatial resolution. Environ Sci Technol. 2016;50: 4742-51. 